\title{
МЕТОД РЕШЕНИЯ МАТРИЧНЫХ УРАВНЕНИЙ, ИСПОЛЬЗУЕМЫХ ДЛЯ СИНТЕЗА СЛОЖНЫХ СПЕЦИАЛЬНЫХ СИСТЕМ
}

\author{
М. В. Павловский \\ ВУНЦ ВВС «Военно-воздушная академия им. проф. Н. Е. Жуковского и Ю. А. Гагарина» (г. Воронеж)
}

Поступила в редакцию 04.12.2017 г.

\begin{abstract}
Аннотация. В статье для ранее обоснованной задачи синтеза сложных специальных систем предложен ряд последовательных преобразований, позволяющих неформализованную до настоящего времени задачу синтеза свести к задаче целочисленного программирования, для которой применим известный алгоритм Краснера, для которого сформированы замечания, отличающие общематематическую задачу решения целочисленного программирования от задачи, решаемой при синтезе сложных специальных систем.

Ключевые слова: сложная специальная система, задача целочисленного линейного программирования, комбинаторный метод.

Annotation. The article for the previously justified to the problem of synthesis of complex special systems proposed a series of successive transformations that allow non-formalized until the present time, the synthesis problem to reduce to the problem of integer programming, for which we apply the well-known Krasner's algorithm for which the generated observations that distinguish the General mathematical problem of solving the integer programming problems solved during the synthesis of complex special systems.

Keywords: special complex system, the problem of integer linear programming, combinatorial method.
\end{abstract}

\section{1. АКТУАЛЬНОСТЬ}

В работах $[1,2]$ для сложных специальных систем (ССС) и образцов специальной техники (ОСТ), их составляющих, приведены основы для применения методов тензорного анализа Крона (ТАК), которые позволят не формализуемую задачу синтеза этих ССС свести к математически обоснованной процедуре. Однако, данные работы, сконцентрированные на обосновании применимости методов ТАК к синтезу ССС, не позволяют автоматически достичь целей синтеза. Целью настоящей статьи является формирование метода и алгоритма решения матричных неравенств, позволяющих с использованием матричного описания ОСТ синтезировать рациональные ССС.

В основе применения методов ТАК к процессу синтеза ССС $[1,2]$ лежит геометриче-

() Павловский М. В., 2018 ское преобразование, позволяющее заменить ОСТ элементами радиотехнических цепей, а всю ССС - системой полосовых и режекторных фильтров, в которой добротность системы эквивалентна эффективности, а полоса пропускания (режекции) фильтра соответствует диапазону рабочих частот этой системы. Это позволяет представить процесс синтеза ССС [1] в виде алгебраического неравенства (1), на которое накладывается система матричных неравенств (2) и ограничений (3), в которых

переменные $i_{1}, i_{2}, \ldots, i_{\text {No }}, j_{1}, j_{2}, \ldots, j_{N k}, b_{1}$, $b_{2}, \ldots, b_{N s}$ определяют наличие в составе ССС, соответственно, $N_{o}$ ОСТ самостоятельного применения, $N_{k}$ комплексов взаимосвязанных алгоритмически и физически ОСТ (КОСТ), $N_{s}$ систем взаимосвязанных алгоритмически и физически ОСТ и КОСТ (СОСТ), показатели эффективности $\mathrm{E}_{O-q}, \mathrm{E}_{K-q}$, $\mathrm{E}_{S-q}$ являются расчетными значениями и $\mathrm{E}_{T R}$ требованиями заказчика к элементам ССС, в 


\section{М. В. Павловский}

качестве которых рассматриваются ОСТ самостоятельного применения, КОСТ, СОСТ из состава этой ССС, связанные единством управления, целей и задач,

коэффициенты при переменных представляют собой матрицы $\mathbf{A}_{\mathbf{0 - 1}}, \mathbf{A}_{\mathbf{0}-2}, \ldots$, $\mathbf{A}_{\mathbf{0}-N o}, \quad \mathbf{A}_{K-1}, \quad \mathbf{A}_{K-2}, \ldots, \quad \mathbf{A}_{K-N k}, \quad \mathbf{A}_{S-1}, \quad \mathbf{A}_{S-2}, \ldots$, $\mathbf{A}_{S-N s}$, которые описывают, соответственно, No ОСТ самостоятельного применения, $N_{k}$ КОСТ, $N_{s}$ СОСТ из состава ССС, связанные единством управления, целей и задач,

матрицы $\mathbf{E}_{\mathbf{O}-\mathbf{T r}}, \mathbf{E}_{\mathbf{K}-\mathbf{T r}}, \mathbf{E}_{\mathrm{S}-\mathrm{Tr}}$ задают ограничения по соответствующим характеристикам, соответственно, ОСТ, КОСТ и СОСТ из состава ССС,

переменные величины $N_{o}, N_{k}, N_{s}$ являются ограничениями по количеству соответственно ОСТ самостоятельного применения, КОСТ, СОСТ из состава ССС.

$$
\begin{gathered}
\sum_{q=1}^{N_{O}} i_{q} \mathrm{E}_{O-q}+\sum_{q=1}^{N_{K}} j_{q} \mathrm{E}_{K-q}+\sum_{q=1}^{N_{S}} b_{q} \mathrm{E}_{S-q} \geq \mathrm{E}_{T R} \\
\left\{\begin{array}{c}
i_{q} \geq 0, j_{q} \geq 0, b_{q} \geq 0 \\
i_{q}, j_{q}, b_{q}-\text { userble }
\end{array}\right. \\
S= \\
=\left\{\begin{array}{ccccc}
i_{1} \mathbf{A}_{\mathbf{O}-1}+ & i_{2} \mathbf{A}_{\mathbf{O}-2}+ & \ldots & i_{N o} \mathbf{A}_{\mathbf{O}-\mathrm{No}} \geq & \mathbf{E}_{\mathbf{O}-\mathrm{Tr}} \\
j_{1} \mathbf{A}_{K-1}+ & j_{2} \mathbf{A}_{K-2}+\ldots & j_{N k} \mathbf{A}_{K-N k} \geq & \mathbf{E}_{\mathrm{K}-\mathrm{Tr}} \\
b_{1} \mathbf{A}_{S-1}+ & b_{2} \mathbf{A}_{S-2}+ & \ldots & b_{N S} \mathbf{A}_{S-N s} \geq & \mathbf{E}_{S-\mathrm{Tr}} \\
i_{1}+ & i_{2}+ & \ldots & i_{N o} \leq & N_{O} \\
j_{1}+ & j_{2}+ & \ldots & j_{N k} \leq & N_{K} \\
b_{1}+ & b_{2}+ & \ldots & b_{N s} \leq & N_{S}
\end{array}\right.
\end{gathered}
$$

В задаче (1) с ограничениями (2) и (3) в качестве ОСТ самостоятельного применения могут рассматриваться средства индивидуальной защиты образцов в составе ССС, отдельные ОСТ, действующие исключительно по собственным алгоритмам функционирования, а также ОСТ, предназначенные для их использования в разведывательно-диверсионных группах. В этих же формулах под КОСТ понимаются совокупности функционально связанных отдельных ОСТ и средств обеспечения их применения, объединенных для выполнения определенных задач. В задаче (1) с ограничениями (2) и (3) в качестве СОСТ понимаются структурно и закономер- но, сбалансировано и целесообразно связанные между собой ОСТ, предназначенные для решения задач обеспечения специальной деятельности, а также комплексы и средства обеспечения их применения и эксплуатации. В задаче (1) с ограничениями (2) и (3) в качестве СОСТ из состава ССС подразумеваются структуры, состоящие в отдельных подразделениях в составе ССС.

Для решения задачи (1) с ограничениями (2) и (3) в исследовании операций [3] предусмотрен раздел линейного программирования. Обычно в задачах линейного программирования не требуется, чтобы коэффициенты при переменных были целыми числами. Однако в практике (особенно при синтезе ССС) часто приходится сталкиваться с задачами, в которых эти коэффициенты должны быть целыми числами, и такие задачи называются задачами целочисленного программирования. При решении задач линейного программирования графическим методом и симплекс-методом нет гарантий, что полученные коэффициенты будут целыми числами. В некоторых случаях допускается округление результатов. Например, если в оптимальном плане предусмотрено, что для формирования ССС следует затратить 499 683,3 условных единицы, то экономически обосновано округление результата до 499683 или даже до 500000 условных единиц. Однако, существуют задачи обоснования ССС, в которых подобное округление может создать большую ошибку. Например, если при решении задачи линейного программирования получено, что следует создать 0,67 СОСТ, то формальное округление до 0 или 1 недопустимо.

Поэтому большое практическое значение имеют методы решения задач линейного программирования, с помощью которых можно найти оптимальный план, координаты которого - целые числа. Задачи целочисленного программирования решаются именно такими методами. Однако на практике такие задачи, в которых коэффициенты при переменных представляют собой матрицы, до настоящего времени не встречались, поэтому решение задачи (1) с ограничениями (2) и (3) является не тривиальной задачей, а для на- 
хождения решения предлагается метод, основанный на отдельном (для элементов матриц $\mathbf{A}_{\mathbf{O}-1}, \mathbf{A}_{\mathbf{O}-2}, \ldots, \mathbf{A}_{\mathbf{O}-N o}, \mathbf{A}_{K-1}, \mathbf{A}_{K-2}, \ldots, \mathbf{A}_{K-N k}$, $\mathbf{A}_{s-1}, \mathbf{A}_{s-2}, \ldots, \mathbf{A}_{s-N s}$ и $\left.\mathbf{E}_{\mathbf{O}-\mathrm{Tr}}, \mathbf{E}_{\mathrm{K}-\mathrm{Tr}}, \mathbf{E}_{\mathrm{S}-\mathrm{Tr}}\right) \mathrm{pe}-$ шении задач целочисленного программирования и нахождении на этой основе значений переменных $i_{1}, i_{2}, \ldots, i_{N o}, j_{1}, j_{2}, \ldots, j_{N k}, b_{1}$, $b_{2}, \ldots, b_{N s}$, обращающих выражение (1) в максимум и удовлетворяющих выражению (2).

Таким образом, в данном разделе показана актуальность и необходимость разработки метода и алгоритма решения матричных неравенств, позволяющих с использованием матричного описания ОСТ синтезировать рациональные ССС.

\section{2. ОБЩИЕ ПОЛОЖЕНИЯ}

В литературе (например, в [4]) приведены численные методы решения матричных уравнений, которые основаны на предварительном приведении исследуемой матрицы к специальной форме, так называемой форме Шура. Несмотря на то, что процедура приведения матриц к форме Шура в настоящее время является хорошо отработанной вычислительной процедурой [4], в интересах синтеза ССС излагаемые в [4] методы решения матричных уравнений не могут быть использованы по причинам:

1. Необходимо вычисление транспонированных и ортогональных матриц, что, требуя сопоставимых с исходной задачей вычислительных мощностей, увеличивает стоимость и время вычисления ответа задачи решения матричного уравнения. Такое положение становится особенно неприемлемым в условиях строгих временных и финансовых ограничений.

2. Основные этапы предлагаемого в [4] алгоритма в каждом случае используют ортогональные преобразования, что также приводит к возрастанию необходимых временных ресурсов.

Поэтому в настоящей работе предлагается оригинальный метод решения матричных уравнений, в основе которого лежит замена системы матричных уравнений на систему линейных уравнений, для решения которой могут быть использованы стандартные алгоритмы без дополнительных преобразований на этапе решения.

В соответствии с предлагаемым методом система неравенств (3) преобразуется в систему неравенств (4), в которой используются только числа. При этом, в соответствии с данными [1], каждая матрица $\mathbf{A}_{\mathbf{o - 1}}, \mathbf{A}_{\mathbf{0 - 2}}, \ldots$, $\mathbf{A}_{\mathbf{O}-\mathrm{No}}, \mathbf{A}_{K-1}, \mathbf{A}_{K-2}, \ldots, \mathbf{A}_{K-N k}, \mathbf{A}_{S-1}, \mathbf{A}_{S-2}, \ldots, \mathbf{A}_{S-N s}$, $\mathbf{E}_{\mathbf{O}-\operatorname{Tr}}, \mathbf{E}_{\mathbf{K}-\operatorname{Tr}}, \mathbf{E}_{\mathrm{S}-\mathrm{Tr}}$ размером $5 \times 5$ представляется двадцатью пятью уравнениями.

$$
\begin{aligned}
& S= \\
& =\left\{\begin{array}{ccccc}
i_{1} a_{\mathbf{O}-\mathbf{1}_{11}}+ & i_{2} a_{\mathbf{O}-2_{11}}+ & \ldots & i_{N o} a_{\mathbf{O}-N o_{11}} \geq & e_{\mathbf{O}-\mathrm{Tr}_{11}} \\
i_{1} a_{\mathbf{O}-\mathbf{1}_{12}}+ & i_{2} a_{\mathbf{O}-2_{12}}+ & \ldots & i_{N o} a_{\mathbf{O}-\mathrm{No}_{12}} \geq & e_{\mathbf{O}-\mathrm{Tr}_{12}} \\
\ldots & \ldots & \ldots & \ldots & \ldots \\
i_{1} a_{\mathbf{O}-\mathbf{1}_{21}}+ & i_{2} a_{\mathbf{O}-2_{21}}+ & \ldots & i_{N o} a_{\mathbf{O}-N o_{21}} \geq & e_{\mathbf{O}-\mathrm{Tr}_{21}} \\
\ldots & \ldots & \ldots & \ldots & \ldots \\
j_{1} a_{K-\mathbf{1}_{11}}+ & j_{2} a_{K-2_{11}}+ & \ldots & j_{N k} a_{K-N k_{11}} \geq & e_{\mathbf{K}-\mathrm{Tr}_{11}} \\
\ldots & \ldots & \ldots & \ldots & \ldots \\
b_{1} a_{S-\mathbf{1}_{55}}+ & b_{2} a_{S-2_{55}}+ & \ldots & b_{N s} a_{S-N N_{55}} \geq & e_{S-\mathrm{Tr}_{55}} \\
i_{1}+ & i_{2}+ & \ldots & i_{N o} \leq & N_{O} \\
j_{1}+ & j_{2}+ & \ldots & j_{N k} \leq & N_{K} \\
b_{1}+ & b_{2}+ & \ldots & b_{N s} \leq & N_{S}
\end{array}\right.
\end{aligned}
$$

Система неравенств (4) требует значительного вычислительного ресурса, однако ее решение позволит:

1) из предлагаемых перспективных структур ССС исключить волюнтаристские структуры, что приведет к сокращению времени обоснования неприемлемых ССС;

2) математически точно обосновать структуры данных ССС, что позволит лицам, принимающим решение, наиболее полно учесть известные исходные данные и незавысить возможности ССС;

3) обеспечить выполнение ограничений заказчика по уровню организационно-штатной структуры ССС и наличию в ней транспортных единиц.

Как уже указывалось, задача (1) с ограничениями (2) и (4) относится к разряду задач целочисленного программирования (ЗЦЛП). К настоящему времени существует достаточно много алгоритмов решения ЗЦЛП. Все методы можно условно разбить на три основные группы: методы отсечения, комбинаторные 


\section{М. В. Павловский}

методы и приближенные методы [5]. Методы отсечений при всей своей теоретической обоснованности (наиболее известный из них метод Гомори) зачастую плохо реализуемы даже на современных компьютерах. Это связано с чрезвычайно малыми отсечениями и накоплением ошибок вычислений. Кроме того, как отмечалось в [6] , можно утверждать, что количество опубликованных по этим методам работ существенно превосходит число решенных этими методами задач. Основным недостатком методов отсечений является то, что они используют нецелочисленную идеологию для решения целочисленных задач.

Комбинаторные методы [5] основаны на целочисленной (дискретной) идеологии и реализуются в виде различных схем направленного перебора. Недостатком большинства из них является отсутствие условий остановки, что может привести к большому и даже к полному перебору. При этом комбинаторные методы достаточно хороши для получения приближенных решений, что особенно важно при дефиците стоимостных и временных ресурсов.

Применение методов приближенного решения задач целочисленного линейного программирования наиболее целесообразно при нахождении решений, которые носят текущий, оперативный характер [7]. При этом более ценным является получение быстрого и гарантированного решения, пусть даже носящего приблизительный характер. Приближенные методы решения задач целочисленного линейного программирования могут быть решены с использованием методик случайного поиска или приемов эвристического анализа. Это не гарантирует получения искомого решения ЗЦЛП.

Таким образом, представленный анализ методов решения ЗЦЛП показывает предпочтительность комбинаторных методов, что определяет решение представленной задачи методом ветвей и границ, наиболее часто используемым комбинаторным методом решения задач целочисленного программирования.

\section{3. ОПИСАНИЕ АЛГОРИТМА}

Для решения представленной задачи сформирован алгоритм, позволяющий непротиворечиво сформировать систему неравенств (3), осуществить корректное сложение и умножение коэффициентов, а также проводить процесс решения задачи целочисленного программирования методом ветвей и границ. На первом этапе осуществляется описание всех ОСТ, КОСТ и СОСТ в составе ССС в виде отдельных матриц, элементами которых являются тактико-технические характеристики.

На втором этапе производится избавление от единиц измерения всех характеристик с помощью стандартных методов нормализации [8].

На третьем этапе нормализованные характеристики приводятся к единому виду стимуляторов [8], что позволит обеспечить единое влияние всех характеристик на результирующий показатель: увеличение каждой отдельной характеристики (независимо от того, что анализируем ОСТ, КОСТ или СОСТ) будет способствовать улучшению всей ССС.

На четвертом этапе определяются ограничения по количеству ОСТ самостоятельного применения (NO), КОСТ (NK), СОСТ (NS) и ограничения $e_{\hat{\mathrm{I}}-\mathrm{Tr}}, e_{\mathrm{K}-\mathrm{Tr}}, e_{\mathrm{S}-\mathrm{Tr}}$ по соответствующим характеристикам.

На пятом этапе сформированная стандартная ЗЦЛП решается стандартным методом ветвей и границ, например, по общеизвестным алгоритмам [9] или по алгоритму Краснера [5].

В отличие от ЗЦЛП, решаемой по алгоритму Краснера [5], в задаче синтеза ССС отрицательные составляющие, учитываемые в выражениях (1), (2) и (4), не рассматриваются, так как это противоречит физической сущности ОСТ, КОСТ и СОСТ, следовательно, вводимое в [5] достаточно большое число $\mu$, отрицательное значение которого ограничивает решение ЗЦЛП снизу, в нашей задаче вырождается в ноль.

Сделаем несколько важных замечаний отличающих задачу, решаемую в [5], от задачи, решаемой при синтезе ССС: 
1. Заданная ЗЦЛП при синтезе ССС не может решаться в интересах минимизации выражения (1), так как мы стремимся к максимизации эффективности своей системы, а не к минимизации эффекта противостоящей CCC.

2. Согласно сделанному замечанию в задаче синтеза ССС отрицательные составляющие, учитываемые в выражениях (1), (2) и (4), не рассматриваются, что позволяет отказаться от замечания № 3 в [5].

При решении задачи синтеза рациональной ССС возникает проблема останова перебора, что может привести к большому и даже к полному перебору возможных структур ССС. Данная проблема становится особенно чувствительной в условиях анализа большого числа ОСТ, КОСТ и СОСТ, составляющих основу ССС. Возможен случай, когда стремление к возрастанию целевой функции (1) при движении по анализируемым ветвям либо приводит к той же узловой точке (то есть зацикливанию алгоритма), либо приводит к незначительному росту целевой функции (1) при одновременном и несопоставимом возрастании потребления ресурсов.

Если мы вернулись к той же точке, то ищутся те координаты (определяющие движение по новым ветвям), по которым ни разу не было движения, и выполняется движение по ним, начиная с максимального полученного значения. Если вновь произошел возврат в прежнюю точку и все не проверенные координаты вырождаются в пустые множества, то найденная точка - искомое решение ЗЦЛП при синтезе ССС, состоящей из ОСТ, КОСТ и СОСТ. Если «зацикливания» не происходило, то дополнительных ходов больше нет и все индексные множества пусты, то найденная точка также является решением ЗЦЛП при синтезе ССС, состоящей из ОСТ, КОСТ и СОСТ.

Для принятия решения об останове при обнаружении незначительного роста целевой функции (1) при одновременном и несопоставимом возрастании потребления ресурсов достаточно применения разработанных методов специального экономического анализа, разработанных автором под руковод- ством профессора доктора технических наук Луценко А.Д.

Таким образом, осуществлено описание алгоритма решения сформированной ЗЦЛП, используемой при синтезе ССС, содержащих ОСТ, КОСТ и СОСТ, и сформулированы замечания, которые отличают задачу, решаемую в [5], от задачи, решаемой при синтезе ССС. Кроме того, в данном разделе определен порядок действий, обеспечивающий останов перебора по методу ветвей и границ.

\section{4. ПОРЯДОК ИСПОЛЬЗОВАНИЯ МЕТОДА. СВЕДЕНИЯ ОБ АПРОБАЦИИ}

В качестве исходных данных для решения задачи синтеза ССС, содержащих ОСТ, КОСТ и СОСТ рассматриваются:

1) задачи, возлагаемые на ССС, которые содержат ОСТ, КОСТ и СОСТ, которые определяют требования по эффективности, учитываемые в (4);

2) состав и структура ССС, содержащих ОСТ, КОСТ и СОСТ, которые могут быть определены по данным имитационного моделирования [10];

3) оценки эффективности ССС в целом и ОСТ, КОСТ и СОСТ их составляющих, используемые в формулах (3)-(4);

4) потребные и достижимые оценки прироста эффективности ОСТ, КОСТ, СОСТ и ССС в целом, получаемые по данным имитационного моделирования [10].

Порядок использования метода представлен в виде вышеописанных этапов. Ограничения и допущения, существенные для применения метода и вытекающие из его назначения и цели, определяют анализ действий ССС с последовательным снижением от ее целей через цели действий СОСТ, через цели действий КОСТ, до задач ОСТ.

Для проверки работоспособности метода были проведены предварительные расчеты, потому что проведение детальных расчетов наталкивается на необходимость использования всего перечня исходных данных, представленных выше. В настоящее время данные оценки не получены из-за трудностей экспе- 


\section{М. В. Павловский}

риментальной проверки ССС. Данные предварительных расчетов показали:

1. В отличие от существующих методов синтеза, основанных на переборе возможных состава и структур ССС, предлагаемый метод, требуя большего перечня исходных данных, обеспечивает сокращение необходимого времени не менее чем в два раза, причем с понижением уровня применения ССС данный показатель возрастает до пяти-шести.

2. В отличие от существующих методов синтеза ССС предлагаемый метод исключает принятие волюнтаристских решений вследствие обоснования четких правил останова процесса решения ЗЦЛП.

3. Применение в предлагаемом методе основных системо-технических принципов и положений [11] позволит сократить количество анализируемых ветвей, что позволит сократить потребное время для синтеза рациональных ССС, содержащих ОСТ, КОСТ и COCT.

Таким образом, предложен метод и алгоритм решения матричных неравенств (1)-(4) на основе решения задачи целочисленного линейного программирования, позволяющие с использованием матричного описания ОСТ, КОСТ и СОСТ осуществлять непротиворечивый и математически обоснованный синтез ССС. В отличие от известных методов и алгоритмов предложенная процедура позволяет: 1) исключить не формализуемые методы экспертного подхода; 2) математически точно и строго обосновать структуры перспективных CCC; 3) обеспечить выполнение ограничений заказчика по различным видам потребных ресурсов. Следовательно, цель настоящей статьи достигнута.

\section{СПИСОК ЛИТЕРАТУРЫ}

1. Павловский, М. В. Модель применения тензорного анализа Крона в интересах синтеза сложных специальных систем радиоэлектронного вооружения Сборник докладов на XXII международной научно-технической конференции «Радиолокация, навигация, связь» RLNC-2016. - Воронеж : НПФ «САКВОЕЕ», 19-21 апреля, 2016. - Т. 3. - С. 1523-1530.
2. Павловскиц̆, М. В. Геометрическая теория иерархических построений сложных специальных систем радиоэлектронного вооружения Сборник докладов на XXII международной научно-технической конференции «Радиолокация, навигация, связь» RLNC2016. - Воронеж : НПФ «САКВОЕЕ», 19-21 апреля, 2016. - Т. 3. - С. 1531-1546.

3. Исследование операций в экономике. Учебное пособие для вузов / Н. Ш Кремер, Б. А. Путко, И. М. Тришин, М. Н. Фридман; под ред. Проф. Н.Ш. Кремера. - М. : ЮНИТИ, 2001. - 407 c.

4. Икрамов, Х. Д. Численное решение матричных уравнений. Под редакцией Д. К. Фадеева. - М. : Наука. Главная редакция физико-математической литературы, 1984. - 192 с.

5. Краснер Н. Я., Пастухов А. И., Щепина И. Н. Алгоритм решения задачи целочисленного линейного программирования. URL: http://pandia.ru/text/77/476/7028.php

6. Модели и методы оптимизации / Отв. ред. Э. Р. Смольяков. - М. : ВНИИ системных исследований, 1991 (1992). - 87 с.

7. Ситникова, О. Д. разработка и исследование некоторых методов решения задач целочисленного линейного программирования общего и специального видов. Диссертация на соискание ученой степени кандидат физико-математических наук. Специальность 01.01.09 - «Математическая кибернетика». Донецк: Донецкий политехнический институт, 1984.

8. Плюта, В. Сравнительный многомерный анализ в эконометрическом моделировании. Пер. с польского. Библиотека иностранных книг для экономистов и статистиков. - М.: «Финансы и статистика», 1989. - 175 с.

9. Курганова, Н. А. Основные методы решения задач линейного программирования: Учебное пособие. - Омск, 2011.

10. Павловский, М. В. Имитационное моделирование радиоэлектронного конфликта сложных эргатических систем: структура модели. Материалы XV Международной научно-методической конференции «Информатика: проблемы, методология, технологии» 12-13 февраля 2015 года. - Воронеж : Издательский дом ВГУ, 2015. - Т. 1. С. 369-373. 
11. Дружинин В. В., Конторов Д. С. Основы военной системотехники. - М. : МО СССР, войска ПВО. - 1983. - 415 с.

Павловский Максим Викторович - канд. техн. наук, доцент кафедры Боевого применения средств радиоэлектронной борьбы (с наземными системами управления) Военного учебно-научного центра Военно-воздушных сил «Военно-воздушная академия имени профессора Н. Е. Жуковского и Ю. А. Гагарина» (г. Воронеж).

Тел.: 8-915-548-32-39

E-mail: pmv-160570@yandex.ru
Pavlovsky Maxim Viktorovich - Candidate of technical Sciences, associate Professor of the Department of Combat use of electronic warfare (with ground control systems) Military training and research center of the air force «Air force Academy named after Professor N. E. Zhukovsky and Y.A. Gagarin» (Voronezh).

Tel.: 8-915-548-32-39

E-mail:pmv-160570@yandex.ru 